\title{
Prognostic and Safety Roles in Laparoscopic Versus Abdominal Radical Hysterectomy in Cervical Cancer: A Meta-analysis
}

\author{
Tiefeng Cao, MD, ${ }^{*}$ Yanling Feng, MD, ${ }^{*}$ Qidan Huang, MD, Ting Wan, MD, and Jihong Liu, MD, PhD
}

\begin{abstract}
Objective: Studies comparing the prognostic results between laparoscopic radical hysterectomy (LRH) and abdominal radical hysterectomy (ARH) in cervical cancer reported contradictory results. We aimed to evaluate the prognostic and safety roles of LRH by pooling studies in a meta-analysis.

Materials and Methods: Original articles were searched in PubMed, EMBASE, and the Cochrane Library. The survival results (5-year disease-free survival [DFS], 5-year overall survival [OS], and recurrence rate [RR]), safety parameters (intra-, peri-, and postoperative complication rates and postoperative bowel or bladder recovery days), efficiency parameters (pelvic/para-aortic lymph nodes removed), and other parameters (operative time, estimated blood loss, and hospital of stay) between the two approaches were reviewed.

Results: For the 2922 cases identified, DFS, OS, and RR did not differ in balanced prognostic factors, including lymph node metastasis, Stage IIB or above, non-squamous cancer histology, grade G3, lymphovascular space invasion, tumor size $\geq 4 \mathrm{~cm}$, and positive parametrial and vaginal margin rates. Meanwhile, LRH was associated with higher complication rates and a shorter time to the recovery of bowel or bladder function than for ARH. The number of removed pelvic or para-aortic lymph nodes did not significantly differ. Other parameters showed LRH was associated with a longer operative time, less blood loss, and a shorter length of hospital stay. The survival and prognostic results did not differ in balanced prognostic factors.
\end{abstract}

Conclusions: LRH is safe and has lower operative complication rates than ARH.

\section{Introduction}

$\mathbf{F}$ Rench Gynecologist Raoul Palmer was the first to use laparoscopy for minor surgical interventions in the late 1950s; in 1989, Reich described the first laparoscopic hysterectomy. ${ }^{1}$ Laparoscopy offers many advantages, ${ }^{2}$ including less pain, faster recovery, shorter hospital stay, and quicker return to normal activities.

Radical hysterectomy is conventionally used to surgically manage early-stage cervical carcinoma. ${ }^{3}$ The most commonly used definition was proposed by Piver et al. ${ }^{4}$ in 1974 , combining with a pelvic with or without para-aortic lymphadenectomy. ${ }^{5}$ Abdominal radical hysterectomy (ARH) is associated with postoperative morbidities such as bladder dysfunction, colorectal motility disorders, and longer hospital stay. Because of advances in minimally invasive surgery, laparoscopic radical hysterectomy (LRH) is now routinely performed worldwide. The laparoscopic approach has fewer operative complications and less morbidity than the abdominal procedure without lowering the cure rate. ${ }^{6}$

More recently, LRH has been increasingly used for operable cervical carcinoma. Although several studies comparing these two techniques have been reported, whether the benefits of LRH are greater remains uncertain because most are small series with conflicting results. ${ }^{7,8}$ The prognostic results were not reviewed before. We decided to rigorously test this belief to evaluate the efficiency, safety, and prognostic results of LRH compared with ARH.

Sun Yat-sen University Cancer Center, State Key Laboratory of Oncology in South China, Collaborative Innovation Center for Cancer Medicine, Guangzhou, People's Republic of China.

*The first two authors contributed equally to this study.

(C) The Author(s) 2015; Published by Mary Ann Liebert, Inc. This Open Access article is distributed under the terms of the Creative Commons Attribution Noncommercial License (http://creativecommons.org/licenses/by-nc/4.0/) which permits any noncommercial use, distribution, and reproduction in any medium, provided the original author(s) and the source are credited. 


\section{Materials and Methods}

Literature search, inclusion, data extraction, and outcomes of interest

A prospective protocol was prepared according to the Preferred Reporting Items for Systematic Reviews and Metaanalysis ${ }^{9}$ and Meta-analysis of Observational Studies in Epidemiology recommendations. ${ }^{10}$ All related studies published through April 1, 2015 were screened by systematic searches in PubMed, EMBASE, and the Cochrane Library. Medical subject heading terms were identified, and their combinations were searched in [Title/Abstract]:laparoscopy/laparoendoscopy/ peritoneoscopy/abdominoscopy/transabdominal/laparotomic/ open/traditional/abdominal, radical hysterectomy, and uterine cervical neoplasms. The Related Articles function was also used, and reference lists of all retrieved studies, review articles, and conference abstracts were also manually searched. When reports published data on the same population, the most recent report was used.

All available randomized controlled trial (RCTs), nonrandomized controlled designs, and retrospective comparative studies (cohort or case-control studies) were included. Patients had biopsy-proven cervical cancer and had a radical hysterectomy performed. Editorials, letters to the editor, review articles, case reports, and animal experimental studies were excluded.

The primary outcomes were 5-year disease-free survival (DFS), 5-year overall survival (OS), and recurrence rate (RR). The secondary outcomes included the safety parameters, the efficiency parameters, and others. The safety parameters were intraoperative bowel or urinary injury, intraoperative, perioperative, and postoperative complications, and postoperative bladder or bowel recovery days. The efficiency parameters were number of pelvic and para-aortic lymph nodes removed. Other parameters were the operative time, estimated blood loss (EBL), and length of stay (LOS). If sufficient data were available, perioperative complications were subdivided into intraoperative complications and postoperative complications within 30 days of surgery.

Additionally, because prognostic factors are important for determining the survival rate, the characteristics of grade, histology, lymphovascular space invasion, positive lymph node rate, positive parametrial margin rate, and positive vaginal margin rate between the two groups were analyzed.

\section{Quality assessment and statistical analysis}

The quality assessment and statistical analysis were done by the criteria of the Centre for Evidence-Based Medicine in Oxford, United Kingdom, based on the level of evidence provided. ${ }^{11}$ For RCTs, the Cochrane risk of bias tool was used to assess methodological quality. For retrospective studies, the modified Newcastle-Ottawa Scale, ${ }^{12,13}$ comprising three factors (patient selection, study group comparability, and outcome assessment), was used in methodological quality assessment. We allocated a score from 0 to 9 (allocated as stars) to each study (except RCTs) based on their methodological quality assessment results. RCTs and observational studies with six or more stars were considered to be of high quality.

All meta-analyses were performed using Review Manager version 5.0 software (Cochrane Collaboration, Oxford).
Continuous variables were analyzed using the weighted mean difference (WMD), dichotomous variables were analyzed using odds ratios (ORs), and survival variables were compared using hazard ratios (HRs). We reported the results with 95\% confidence intervals (CIs). We calculated the standard deviations of studies that presented continuous data as the means and ranges using the technique described by Hozo et al. ${ }^{14}$ Statistical heterogeneity between studies was assessed using a chi-squared test, with significance set at $P<.10$, as well as using the $I^{2}$ statistic. A random-effects model was used if heterogeneity existed between studies; otherwise, a fixed-effects model was used. Subgroup analyses were performed to define safety and efficiency of the LRH approach. We also performed sensitivity analyses for high-quality studies and used funnel plots to screen for potential publication bias.

\section{Results}

\section{Characteristics of studies}

Twenty-two studies including 2922 cases (1230 LRH cases and 1692 ARH cases) met the inclusion criteria. Twenty-one publications were full-text articles, ${ }^{15-36}$ and one publication was an abstract. ${ }^{37}$ The references listed for these studies and review articles were also examined, but no further studies were included.

Table 1 shows the characteristics of included studies, which included 1 small $\mathrm{RCT}^{15}$ (Level of Evidence 2b), 15 retrospective studies, ${ }^{16-29,37}$ and 6 prospective studies $^{30-33,35,36}$ comparing a contemporary series of patients (Level of Evidence $3 b$ ). Five studies examined cases with Stage IB1 or below disease. ${ }^{15,16,26,32,37}$ Sixteen studies included cases with locally advanced Stage IB2-IIB disease, ${ }^{17-20,22-25,27-30,34-36}$ and only two studies ${ }^{31,33}$ included cases with Stage IIB disease. One study defined stage IB1 as a tumor size of less than $2 \mathrm{~cm} .{ }^{32}$ One study included cervical cancer tumors diagnosed as FIGO Stage IB-IIA, with a tumor diameter of $3 \mathrm{~cm}$ or greater. ${ }^{18}$ Patients in one study were treated with neoadjuvant chemotherapy prior to radical surgery. ${ }^{33}$ Outcomes are shown in Table 2.

\section{Methodological quality of the included studies}

The quality of the included studies was generally low. Only one RCT was included. Matching criteria between the groups varied, and little matching information was identified from the conference abstracts. Twenty-one studies mentioned the length of follow-up time, except for one abstract lacking the full article ${ }^{37}$; the follow-up time varied in these studies.

\section{Primary outcomes}

Among these studies, prognostic factors showed no significant difference, including Stage IIB or above (only two studies included Stage IIB or above $\mathrm{e}^{23,31}$ and showed no differences; others included cases below Stage IIB), Grade G3 $(\mathrm{OR}=1.44 ; 95 \% \mathrm{CI}, 0.70,2.96 ; P=.32)$, non-squamous cancer histology $(\mathrm{OR}=0.98 ; 95 \% \mathrm{CI}, 0.78,1.23 ; P=.84)$, positive lymph node rate $(\mathrm{OR}=1.08 ; 95 \% \mathrm{CI}, 0.83,1.41$; $P=.57)$, positive lymphovascular space invasion $(\mathrm{OR}=1.26$; $95 \% \mathrm{CI}, 0.68,2.33 ; P=.47)$, tumor size $\geq 4 \mathrm{~cm}(\mathrm{OR}=1.27$; $95 \% \mathrm{CI}, 0.62,2.62 ; P=.52)$, positive parametrial margin rate 
Table 1. Characteristics of Included Studies

\begin{tabular}{|c|c|c|c|c|c|c|c|c|}
\hline \multirow[b]{2}{*}{ Reference } & \multirow{2}{*}{$\begin{array}{l}\text { Level of } \\
\text { evidence }\end{array}$} & \multirow[b]{2}{*}{ Design } & \multirow[b]{2}{*}{ Stage } & \multicolumn{2}{|c|}{$\begin{array}{c}\text { Number of } \\
\text { patients }\end{array}$} & \multirow[b]{2}{*}{ Matching $^{\mathrm{a}}$} & \multirow{2}{*}{$\begin{array}{c}\text { Follow-up } \\
(\text { months }) \\
(\text { LRH/ARH })^{\mathrm{b}}\end{array}$} & \multirow[b]{2}{*}{ q score } \\
\hline & & & & $L R H$ & $A R H$ & & & \\
\hline Bogani et al. ${ }^{31}$ & $3 b$ & $\mathrm{RP}$ & IA2-IIB & 65 & 65 & $1,3-6,8,9$ & $58.8 / 106.2$ & 7 \\
\hline Campos et al. ${ }^{15}$ & $2 \mathrm{~b}$ & RCT & IA2-IB1 & 16 & 14 & $1,2,4,5,7,8,10$ & 5 years/5 years & $\mathrm{RCT}$ \\
\hline Diaz-Feijoo et al. ${ }^{22}$ & $3 \mathrm{~b}$ & $\mathrm{R}$ & IA2-IIA & 20 & 30 & $4,5,8$ & $22.5 / 35$ & 7 \\
\hline Ditto et al. ${ }^{32}$ & $3 b$ & RP & IA2-IB1 & 60 & 60 & $1,3-6,8$ & $31 / 48.7$ & 7 \\
\hline Frumovitz et al. ${ }^{17}$ & $3 \mathrm{~b}$ & $\mathrm{R}$ & IA1-IB2 & 35 & 44 & $1,3-5$ & $7.2 / 15.2$ & 7 \\
\hline Ghezzi et $\mathrm{al}^{33^{\circ}}$ & $3 b$ & RP & IB2-IIB & 68 & 273 & $1,3-5$ & $35 / 41$ & 7 \\
\hline Ghezzi et al. ${ }^{23}$ & $3 b$ & $\mathrm{R}$ & IA2-IIA & 50 & 48 & $1-6,8$ & $58 / 10$ & 6 \\
\hline Kong et al. 18 & $3 \mathrm{~b}$ & $\mathrm{R}$ & IB-IIA & 40 & 48 & $1,3-5,7,8$ & $28 / 58$ & 7 \\
\hline Lee et al. ${ }^{24}$ & $3 b$ & $\mathrm{R}$ & I-IIA & 8 & 16 & $1,3-5,8,10$ & $78 / 75$ & 5 \\
\hline Li et al. ${ }^{25}$ & $3 b$ & $\mathrm{R}$ & IB-IIA & 90 & 35 & $1,3,6,9,10$ & $26 / 26$ & 4 \\
\hline Lim et al. & $3 b$ & $\mathrm{RP}$ & IA-IB2 & 18 & 30 & $1-5,9$ & $37.3 / 23$ weeks & 7 \\
\hline Malzoni et al. ${ }^{26}$ & $3 b$ & $\mathrm{R}$ & IA $1-I B 1$ & 65 & 62 & $1,3-5,9$ & $52.5 / 71.5$ & 6 \\
\hline Nam et al. ${ }^{35}$ & $3 b$ & $\mathrm{RP}$ & IA2-IIA & 263 & 263 & $1,4,5,9$ & $92 / 63$ & 7 \\
\hline Park et al. ${ }^{19}$ & $3 b$ & $\mathrm{R}$ & IA2-IIA2 & 99 & 159 & $1-8$ & $49 / 57$ & 3 \\
\hline Park et al. ${ }^{27}$ & $3 b$ & $\mathrm{R}$ & IA2-IIA2 & 54 & 112 & $1-5,7,8$ & $49.2 / 60.9$ & 3 \\
\hline Park et al. ${ }^{28}$ & $3 b$ & $\mathrm{R}$ & IB2, IIA2 & 115 & 188 & $1-5,7-9$ & $30 / 30$ & 6 \\
\hline Sobiczewski et al. ${ }^{20}$ & $3 \mathrm{~b}$ & $\mathrm{R}$ & IA-IIA & 22 & 58 & $1,4-6,9$ & $26 / 47$ & 4 \\
\hline Stanciu et al. & $3 \mathrm{~b}$ & $\mathrm{R}$ & IA2-IB1 & 31 & 45 & NA & NA & 4 \\
\hline Taylor et al. ${ }^{i 6}$ & $3 \mathrm{~b}$ & $\mathrm{R}$ & IA2-IB1 & 9 & 18 & $1,3-5$ & $35.3 / 35.1$ & 6 \\
\hline Toptas and Simsek ${ }^{21}$ & $3 b$ & $\mathrm{R}$ & IA2-IB1 & 22 & 46 & $1,5,9$ & $42.5 / 43.5$ & 5 \\
\hline Uccella et al. ${ }^{29}$ & $3 b$ & $\mathrm{R}$ & IA2-IIA & 50 & 48 & $1-4,8$ & NA & 7 \\
\hline Zakashansky et al. $^{36}$ & $3 b$ & RP & IA1-IIA & 30 & 30 & $1,4-6,9$ & $20 / 40$ & 5 \\
\hline
\end{tabular}

${ }^{\text {a }}$ Matching: 1 =age; 2 =parity; 3 =body mass index; $4=$ stage; $5=$ histology; $6=$ grade; $7=$ American Society of Anesthesiologists score; $8=$ previous abdominal surgery history; $9=$ tumor size; $10=$ single surgeon

'Mean or median.

ARH, abdominal radical hysterectomy; LRH, laparoscopic radical hysterectomy; NA, data not available; R, retrospective; RP, retrospective design, prospective data collection; RCT, randomized controlled trial.

Table 2. Results of Meta-analysis Comparison of Laparoscopic Radical Hysterectomy and AbDominal Radical Hysterectomy

\begin{tabular}{|c|c|c|c|c|c|c|c|c|c|}
\hline \multirow[b]{2}{*}{ Outcomes of interest } & \multirow{2}{*}{$\begin{array}{l}\text { Number } \\
\text { of studies }\end{array}$} & \multicolumn{2}{|c|}{$\begin{array}{c}\text { Number } \\
\text { of patients }\end{array}$} & \multirow[b]{2}{*}{ WMD/OR $(95 \%$ CI) } & \multirow[b]{2}{*}{$\mathrm{P}$ value $\mathrm{a}^{\mathrm{a}}$} & \multicolumn{3}{|c|}{$\begin{array}{c}\text { Study } \\
\text { heterogeneity }\end{array}$} & \multirow[b]{2}{*}{$\mathrm{P}$ value } \\
\hline & & $L R H$ & $A R H$ & & & $\chi^{2}$ & df & $I^{2}(\%)$ & \\
\hline \multicolumn{10}{|l|}{ Primary outcomes } \\
\hline 5-year DFS ${ }^{\mathrm{b}}$ & 10 & 791 & 1031 & $0.01(-0.10,0.11)$ & 91 & 4.78 & 9 & 0 & .85 \\
\hline 5 -year $\mathrm{OS}^{\mathrm{b}}$ & 6 & 656 & 847 & $-0.02(-0.14,0.10)$ & .73 & 4.28 & 5 & 0 & .51 \\
\hline Recurrence rate ${ }^{\mathrm{b}}$ & 13 & 924 & 1350 & $0.82(0.61,1.11)$ & .20 & 3.37 & 12 & 0 & .99 \\
\hline \multicolumn{10}{|l|}{ Secondary outcomes } \\
\hline $\begin{array}{l}\text { Intraoperative } \\
\text { bowel-urinary injury }\end{array}$ & 17 & 1083 & 1282 & $1.50(0.99,2.26)$ & .06 & 6.79 & 16 & 0 & .98 \\
\hline Perioperative complication & 4 & 315 & 555 & $0.56(0.36,0.90)$ & .02 & 2.50 & 3 & 0 & .47 \\
\hline Postoperative complication & 18 & 1078 & 1451 & $0.75(0.62,0.91)$ & .003 & 40.31 & 16 & 60 & .0007 \\
\hline Intraoperative complication & 10 & 523 & 661 & $1.48(0.75,2.91)$ & .25 & 8.31 & 9 & 0 & .50 \\
\hline Bladder recovery (days) & 3 & 61 & 119 & $-2.48(-5.16,0.19)$ & .07 & 17.46 & 2 & 89 & .0002 \\
\hline Anorectal recovery (days) & 4 & 104 & 123 & $-0.80(-1.16,-0.44)$ & $<.00001$ & 4.65 & 2 & 57 & .1 \\
\hline Pelvic LNs removed & 16 & 813 & 1220 & $-1.44(-4.14,1.27)$ & .3 & 247.44 & 15 & 94 & $<.00001$ \\
\hline Para-aortic LNs removed & 2 & 73 & 78 & $-1.79(-6.39,2.82)$ & .45 & 18.41 & 1 & 95 & $<.0001$ \\
\hline Operation time (minutes) & 17 & 841 & 1383 & $18.76(2.13,35.39)$ & .03 & 184.15 & 16 & 91 & $<.00001$ \\
\hline Length of stay (days) & 16 & 1054 & 1437 & $-4.36(-5.38,-3.34)$ & $<.00001$ & 141.26 & 15 & 89 & $<.00001$ \\
\hline Blood loss $(\mathrm{mL})$ & 17 & 1089 & 1481 & $-193.61(-236.80,-150.43)$ & $<.00001$ & 80.44 & 16 & 80 & $<.00001$ \\
\hline
\end{tabular}

${ }^{\text {a }}$ Statistically significant results are shown in bold.

${ }^{\mathrm{b}}$ Prognostic factors were not significantly different.

$\mathrm{ARH}$, abdominal radical hysterectomy; CI, confidence interval; $d f$, degrees of freedom; DFS, disease-free survival; LN, lymph node; LRH, laparoscopic radical hysterectomy; OS, overall survival; WMD/OR, weighted mean difference/odds ratio. 
$(\mathrm{OR}=1.12 ; 95 \% \mathrm{CI}, 0.71,1.78 ; P=.62)$, and positive vaginal margin rate $(\mathrm{OR}=2.79 ; 95 \% \mathrm{CI}, 0.88,8.82 ; P=.08)$.

Under the balanced prognostic factors, the total effect of DFS and OS showed no difference $(\mathrm{HR}=-0.01 ; 95 \% \mathrm{CI}$, $-0.08,0.07 ; P=.88)$. Ten studies ${ }^{18-20,24,26-28,31,32,35}$ including 1822 patients assessed DFS, and six studies assessed the 5-year OS. ${ }^{19,27,28,31,32,35}$ Only two studies ${ }^{31,32}$ calculated HRs. All others included $P$ values and the number of events. No significant differences in 5-year DFS and OS were found between the two groups $(\mathrm{HR}=0.01 ; 95 \% \mathrm{CI},-0.10,0.11$; $P=.91$; and $\mathrm{HR}=-0.02 ; 95 \% \mathrm{CI},-0.14,0.10 ; P=.73$, respectively) (Fig. 1a).

Thirteen studies ${ }^{18-21,24-28,30,32,33,35}$ assessed RR among 2274 patients. During the follow-up period, the RR did not differ between the LRH and ARH groups under the balanced prognostic factors $(8.77 \%$ versus $11.93 \%$; OR $=0.82 ; 95 \%$ CI, 0.61, 1.11; $P=.20$ ) (Fig. 1b).

\section{Secondary outcomes}

Safety parameters. LRH was safer. The total effects of intraoperative intestine or urinary injuries and intraoperative, perioperative, or postoperative complications showed the following result: $12.41 \%$ versus $15.15 \%$; OR $=0.82 ; 95 \% \mathrm{CI}$, $0.71,0.96 ; P=.01$. Also, the total effect of postoperative bowel or bladder recovery days showed ARH had a longer recovery time $(\mathrm{WMD}=-1.08$ days; 95\% CI, $-1.67,0.50$; $P=.0003$ ).

Seventeen studies ${ }^{15-20,22,23,25-29,31,32,35,36}$ including a total of 2365 patients described intraoperative bowel or urinary injury and showed some difference between the LRH group and the ARH group, but the difference was not significant (4.99\% versus $3.12 \%$; $\mathrm{OR}=1.50 ; 95 \% \mathrm{CI}, 0.99,2.26$; $P=.06)$. Other intraoperative and postoperative complication rates were available for 10 studies ${ }^{16,17,20,23-25,27,28,31,36}$ and 18 studies, ${ }^{15-20,23-25,27-33,35,36}$ respectively. Intraoperative complication rates, which mainly were due to vessel and nerve injuries, did not significantly differ between the LRH and ARH groups (3.44\% versus $2.27 \%$; $\mathrm{OR}=1.48 ; 95 \% \mathrm{CI}$, $0.75,2.91 ; P=.25)$. Postoperative complication rates were significantly higher in the ARH group than in the LRH group $(25.09 \%$ versus $31.41 \%$; OR $=0.74 ; 95 \% \mathrm{CI}, 0.61$, $0.90 ; P=.002)$. Four studies ${ }^{19,27,28,35}$ reporting perioperative complication rates showed lower rates in the LAH group compared with the ARH group $(8.57 \%$ versus $14.05 \%$; $\mathrm{OR}=0.56 ; 95 \%$ CI, 0.36, 0.90; $P=.02$ ) (Fig. 2).

Three studies ${ }^{20,24,37}$ and three studies, ${ }^{24,26,37}$ respectively, reported the postoperative bladder and anorectal function recovery time. No significant differences were found for the recovery of bladder function $(\mathrm{WMD}=-2.48$ days; $95 \% \mathrm{CI}$, $-5.16,0.19 ; P=.07)$, but a significant difference was found in the number of days for anorectal function recovery (WMD $=-0.80$ days; 95\% CI, $-1.16,-0.44 ; P<.00001)$ (Table 2).

Efficiency parameters. Efficiency parameters included pelvic and para-aortic lymph nodes removed and showed no difference (WMD $=-1.48 ; 95 \% \mathrm{CI},-3.73,0.77 ; P=.20$ ). Sixteen studies ${ }^{16,18,19,21-28,30-33,36}$ reported the number of pelvic lymph nodes removed, and two studies ${ }^{24,26}$ reported the number of para-aortic lymph nodes removed; no significant differences were found between the two groups for either type of node $(\mathrm{WMD}=-1.44 ; 95 \% \mathrm{CI},-4.14,1.27 ; P=.30$ and $\mathrm{WMD}=-1.79 ; 95 \% \mathrm{CI},-6.39,2.82 ; P=.45$, respectively) (Table 2).

Other parameters. In total, 17 studies ${ }^{15,16,18,19,22-28,30-33,35,36}$ including 2224 cases reported the operative time, and 16 studies $^{16,18,19,22-28,30-33,35,36}$ including approximately 2491 patients reported the LOS. The laparoscopic group required a significantly longer operation time $(\mathrm{WMD}=18.76$ minutes; 95\% CI, 2.13, 35.39; $P=.03)$. The abdominal group had a significantly longer $\operatorname{LOS}(\mathrm{WMD}=-4.36$ days; $95 \% \mathrm{CI}$, $-5.38,-3.34 ; P<.00001$ ) (Table 2).

Seventeen studies ${ }^{16-19,22-28,30-33,35,36}$ reported EBL for 2570 patients and showed significantly greater EBL among the ARH group (WMD $=-193.61 \mathrm{~mL} ; 95 \% \mathrm{CI},-236.80$, $-150.43 ; P<.00001$ ) (Table 2).

\section{Sensitivity analysis and publication bias}

Using the modified Newcastle-Ottawa Scale to assess the quality, $1 \mathrm{RCT}^{15}$ and 13 retrospective studies ${ }^{15-18,22,23,26,28-33,35}$ that scored six or more stars were included in a sensitivity analysis (Table 3). No change occurred in the significance of any of the outcomes except for the perioperative complication rates, which did not significantly differ between the LRH and ARH groups $(\mathrm{OR}=0.66 ; 95 \% \mathrm{CI}, 0.32,1.73 ; P=.22)$. The degree of between-study heterogeneity decreased slightly for DFS, OS, RR, intraoperative bowel and urinary injury, and the perioperative complication rate, although the between-study heterogeneity remained statistically significant for the postoperative complication rate, operative time, EBL, LOS, and number of lymph nodes removed. One study was included for the para-aortic lymph node, and no study included the bladder recovery day in the sensitivity analysis.

The funnel plot of the studies that reported intra-, peri-, and postoperative complications showed that almost all studies lie inside the 95\% CI and have an even distribution around the vertical axis, indicating that no obvious publication bias exists.

\section{Discussion}

LRH has become increasingly common in clinical practice, mainly because the procedure is thought to be safe and efficient, potentially reducing postoperative morbidity compared with that of conventional radical hysterectomy. To examine whether this viewpoint is well founded, a systematic review of the literature was performed to compare the prognostic and safety results of LRH and ARH. This metaanalysis of 1 RCT and 21 retrospective studies including 2922 patients showed that LRH was safe and had significantly fewer peri- and postoperative complications, shorter bowel recovery time, reduced intraoperative blood loss, and shorter hospital stay than ARH, even though the operative time was increased. The rates of intraoperative bladder and intestine injuries were increased but were not significantly different. No significant differences were found in 5-year DFS and OS, as well as RR, during follow-up or in the numbers of pelvic or para-aortic lymph nodes removed.

These conclusions are consistent with at least one study that performed a systematic analysis of one RCT of 13 women, of whom 7 had a laparoscopically assisted radical hysterectomy and 6 had ARH. ${ }^{8}$ 
a

\begin{tabular}{|c|c|c|c|c|c|}
\hline Study or Subgroup & Hazard Ratio & SE & Weight & IV. Fixed, $95 \% \mathrm{Cl}$ & IV. Fixed, $95 \% \mathrm{Cl}$ \\
\hline \multicolumn{6}{|l|}{ 16.1.1 DFS } \\
\hline Bogani.G 2014 & -0.211 & 0.4106 & $0.9 \%$ & $-0.21[-1.02,0.59]$ & 7 \\
\hline Ditto.A 2015 & -0.868 & 0.7564 & $0.3 \%$ & $-0.87[-2.35,0.61]$ & \\
\hline Kong.T.W 2014 & 0.05 & 0.217 & $3.3 \%$ & $0.05[-0.38,0.48]$ & \\
\hline Lee.E.J 2011 & 0.027 & 0.264 & $2.2 \%$ & $0.03[-0.49,0.54]$ & \\
\hline Malzoni.M 2009 & 0.195 & 0.184 & $4.6 \%$ & $0.20[-0.17,0.56]$ & \\
\hline Nam.J.H 2012 & 0.061 & 0.09 & $19.4 \%$ & $0.06[-0.12,0.24]$ & \\
\hline Park.J.K(obese) 2012 & -0.073 & 0.177 & $5.0 \%$ & $-0.07[-0.42,0.27]$ & \\
\hline Park.J.Y (stage)2013 & -0.123 & 0.132 & $9.0 \%$ & $-0.12[-0.38,0.14]$ & \\
\hline Park.J.Y(old) 2012 & -0.049 & 0.134 & $8.7 \%$ & $-0.05[-0.31,0.21]$ & \\
\hline $\begin{array}{l}\text { Sobiczewski.P } 2009 \\
\text { Subtotal }(95 \% \mathrm{Cl})\end{array}$ & 0.173 & 0.275 & $\begin{array}{r}2.1 \% \\
55.6 \%\end{array}$ & $\begin{array}{l}0.17[-0.37,0.71] \\
0.01[-0.10,0.11]\end{array}$ & \\
\hline \multicolumn{6}{|c|}{$\begin{array}{l}\text { Heterogeneity: } \mathrm{Chi}^{2}=4.78, \mathrm{df}=9(\mathrm{P}=0.85) ; \mathrm{I}^{2}=0 \% \\
\text { Test for overall effect: } Z=0.11(P=0.91)\end{array}$} \\
\hline \multicolumn{6}{|l|}{ 16.1.2 os } \\
\hline Bogani.G 2014 & -0.462 & 0.472 & $0.7 \%$ & $-0.46[-1.39,0.46]$ & 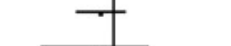 \\
\hline Ditto.A 2015 & -0.691 & 1.028 & $0.1 \%$ & $-0.69[-2.71,1.32]$ & -1 \\
\hline Nam.J.H 2012 & 0.067 & 0.089 & $19.8 \%$ & $0.07[-0.11,0.24]$ & \\
\hline Park.J.K(obese) 2012 & -0.209 & 0.171 & $5.4 \%$ & $-0.21[-0.54,0.13]$ & \\
\hline Park.J.Y (stage)2013 & -0.119 & 0.131 & $9.1 \%$ & $-0.12[-0.38,0.14]$ & \\
\hline $\begin{array}{l}\text { Park.J.Y(old) } 2012 \\
\text { Subtotal }(95 \% \mathrm{Cl})\end{array}$ & 0.042 & 0.13 & $\begin{array}{r}9.3 \% \\
44.4 \%\end{array}$ & $\begin{array}{r}0.04[-0.21,0.30] \\
-0.02[-0.14,0.10]\end{array}$ & \\
\hline \multicolumn{6}{|c|}{ Heterogeneity: $\mathrm{Chi}^{2}=4.28, \mathrm{df}=5(\mathrm{P}=0.51) ; \mathrm{I}^{2}=0 \%$} \\
\hline Total $(95 \% \mathrm{Cl})$ & & & $100.0 \%$ & $-0.01[-0.08,0.07]$ & \\
\hline $\begin{array}{l}\text { Heterogeneity: } \mathrm{Chi}^{2}=\mathrm{S} \\
\text { Test for overall effect: }\end{array}$ & $\begin{array}{l}7, d f=15(P= \\
=0.15(P=0.88\end{array}$ & $0.87) ; 1^{2}=$ & $=0 \%$ & & $\begin{array}{ccccc}-4 & -2 & 0 & 2 & 4 \\
\text { vours lap } & \text { Favours }\end{array}$ \\
\hline
\end{tabular}

Test for subaroun differences: $\mathrm{Chi}^{2}=0.11 . \mathrm{df}=1(\mathrm{P}=0.74) . \mathrm{I}^{2}=0 \%$

b

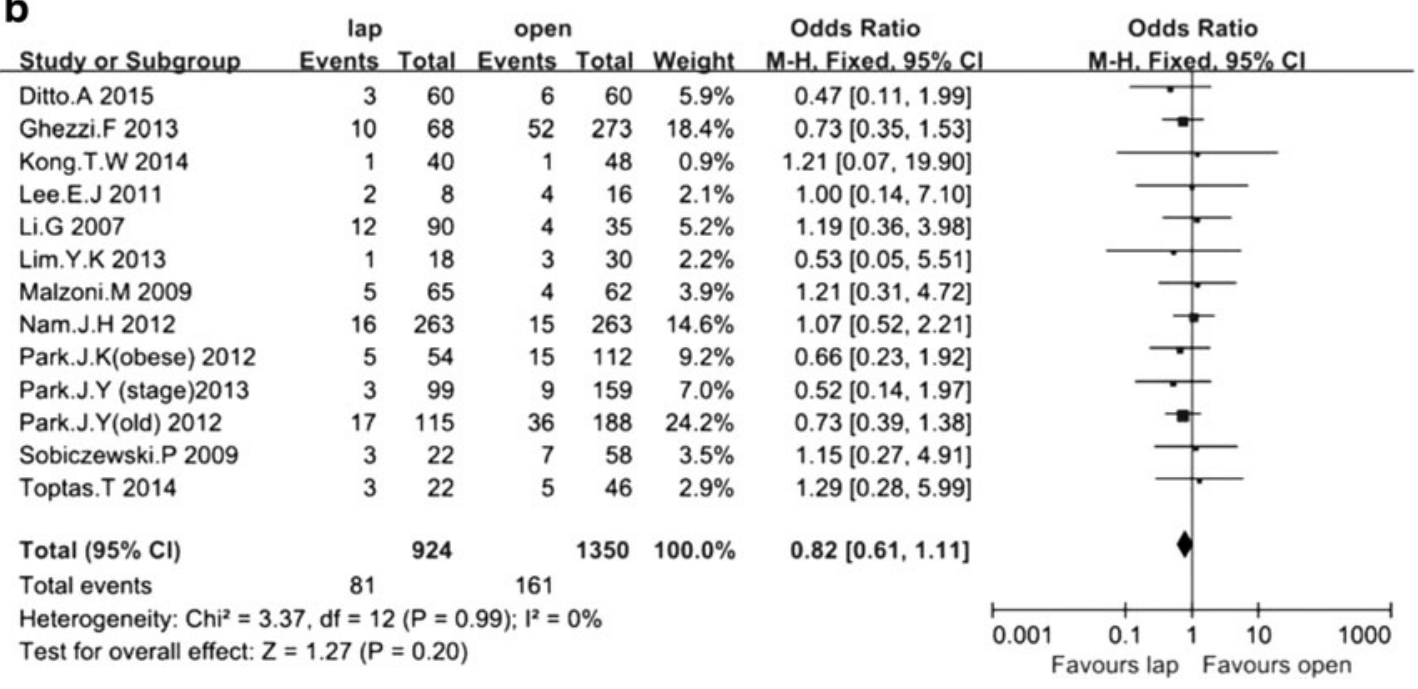

FIG. 1. Forest plot and meta-analysis of prognostic results: (a) 5-year disease-free survival (DFS) and overall survival (OS) and (b) recurrence rate. CI, confidence interval; IV, inverse variance method; lap, laparoscopic; M-H, MantelHaenszel method; SE, standard error; $\mathrm{CI}=$ confidence interval). 


\begin{tabular}{|c|c|c|c|c|c|c|c|c|c|}
\hline \multirow{2}{*}{$\begin{array}{l}\text { Study or Subgroup } \\
10.1 .1 \text { Intra-op AE }\end{array}$} & $\begin{array}{r}\text { lap } \\
\text { Events }\end{array}$ & \multicolumn{2}{|c|}{ open } & \multicolumn{2}{|c|}{ Total Weight } & \multirow[t]{2}{*}{$\begin{array}{c}\text { Odds Ratio } \\
\mathrm{M}-\mathrm{H} \text {, Fixed, } 95 \% \mathrm{Cl}\end{array}$} & \multicolumn{2}{|c|}{$\begin{array}{c}\text { Odds Ratio } \\
\text { M-H, Fixed, } 95 \% \text { C }\end{array}$} & \\
\hline & & & & & & & & & \\
\hline Bogani.G 2014 & 1 & 65 & 1 & 65 & $0.3 \%$ & $1.00[0.06,16.34]$ & & & \\
\hline Frumovitz.M 2007 & 3 & 35 & 1 & 44 & $0.2 \%$ & $4.03[0.40,40.57]$ & & & \\
\hline Ghezzi.F 2007 & 2 & 50 & 5 & 48 & $1.4 \%$ & $0.36[0.07,1.94]$ & & & \\
\hline Lee.E.J 2011 & 2 & 8 & 3 & 16 & $0.4 \%$ & $1.44[0.19,11.04]$ & & & \\
\hline Li.G 2007 & 4 & 90 & 1 & 35 & $0.4 \%$ & $1.58[0.17,14.66]$ & & & \\
\hline Park.J.Y (stage)2013 & 1 & 99 & 2 & 159 & $0.4 \%$ & $0.80[0.07,8.95]$ & & & \\
\hline Park.J.Y(old) 2012 & 1 & 115 & 0 & 188 & $0.1 \%$ & $4.94[0.20,122.26]$ & & & \\
\hline Sobiczewski.P 2009 & 3 & 22 & 0 & 58 & $0.1 \%$ & $21.00[1.04,424.84]$ & & & \\
\hline Taylor.S.E 2011 & 0 & 9 & 2 & 18 & $0.5 \%$ & $0.35[0.02,8.02]$ & & & \\
\hline Zakashansky.K 2007 & 1 & 30 & 0 & 30 & $0.1 \%$ & $3.10[0.12,79.23]$ & & & \\
\hline Subtotal $(95 \% \mathrm{Cl})$ & & 523 & & 661 & $3.9 \%$ & $1.48[0.75,2.91]$ & & & \\
\hline Total events & 18 & & 15 & & & & & & \\
\hline \multicolumn{10}{|c|}{ Heterogeneity: $\mathrm{Chi}^{2}=8.31, \mathrm{df}=9(\mathrm{P}=0.50) ; \mathrm{I}^{2}=0 \%$} \\
\hline Test for overall effect: $z$ & $=1.14(\mathrm{P}$ & $=0.25)$ & & & & & & & \\
\hline 10.1.2 post-op $A E$ & & & & & & & & & \\
\hline Bogani.G 2014 & 4 & 65 & 12 & 65 & $3.2 \%$ & $0.29[0.09,0.95]$ & & & \\
\hline Campos.L.S 2013 & 3 & 16 & 5 & 14 & $1.2 \%$ & $0.42[0.08,2.19]$ & & & \\
\hline Ditto.A 2015 & 2 & 60 & 2 & 60 & $0.5 \%$ & $1.00[0.14,7.34]$ & & & \\
\hline Frumovitz.M 2007 & 35 & 43 & 12 & 44 & $0.6 \%$ & $11.67[4.23,32.19]$ & & & \\
\hline Ghezzi.F 2007 & 7 & 50 & 11 & 48 & $2.7 \%$ & $0.55[0.19,1.56]$ & & & \\
\hline Ghezzi.F 2013 & 29 & 68 & 131 & 273 & $8.4 \%$ & $0.81[0.47,1.38]$ & & & \\
\hline Kong.T.W 2014 & 16 & 40 & 19 & 48 & $2.9 \%$ & $1.02[0.43,2.40]$ & & & \\
\hline Lee.E.J 2011 & 4 & 8 & 7 & 16 & $0.7 \%$ & $1.29[0.23,7.05]$ & & & \\
\hline Li.G 2007 & 36 & 90 & 14 & 35 & $3.4 \%$ & $1.00[0.45,2.22]$ & & & \\
\hline Lim.Y.K 2013 & 2 & 18 & 4 & 30 & $0.7 \%$ & $0.81[0.13,4.95]$ & & & \\
\hline Nam.J.H 2012 & 78 & 263 & 107 & 263 & $21.1 \%$ & $0.61[0.43,0.88]$ & & & \\
\hline Park.J.K(obese) 2012 & 13 & 54 & 40 & 112 & $5.5 \%$ & $0.57[0.27,1.19]$ & $\cdot$ & & \\
\hline Park.J.Y (stage)2013 & 23 & 99 & 56 & 159 & $9.2 \%$ & $0.56[0.32,0.98]$ & $F$ & & \\
\hline Park.J.Y(old) 2012 & 8 & 115 & 28 & 188 & $5.5 \%$ & $0.43[0.19,0.97]$ & $\rightarrow$ & & \\
\hline Sobiczewski.P 2009 & 0 & 22 & 6 & 58 & $1.0 \%$ & $0.18[0.01,3.32]$ & & & \\
\hline Taylor.S.E 2011 & 0 & 9 & 5 & 18 & $1.0 \%$ & $0.13[0.01,2.63]$ & & & \\
\hline Uccella.S 2007 & 10 & 50 & 7 & 48 & $1.6 \%$ & $1.46[0.51,4.22]$ & & & \\
\hline Zakashansky.K 2007 & 6 & 30 & 8 & 30 & $1.8 \%$ & $0.69[0.21,2.30]$ & & & \\
\hline Subtotal $(95 \% \mathrm{Cl})$ & & 1100 & & 1509 & $71.2 \%$ & $0.74[0.61,0.90]$ & & & \\
\hline Total events & 276 & & 474 & & & & & & \\
\hline Heterogeneity: $\mathrm{Chi}^{2}=4$ & $19, \mathrm{df}=1$ & $17(P=0$ & 0.0009 ); । & $\left.\right|^{2}=59 \%$ & & & & & \\
\hline Test for overall effect: $z$ & $=3.11(\mathrm{P}$ & $=0.002$ & & & & & & & \\
\hline 10.1.3 peri-op $\mathrm{AE}$ & & & & & & & & & \\
\hline Nam.J.H 2012 & 3 & 47 & 4 & 96 & $0.7 \%$ & $1.57[0.34,7.31]$ & & & \\
\hline Park.J.K(obese) 2012 & 4 & 54 & 21 & 112 & $3.5 \%$ & $0.35[0.11,1.07]$ & & & \\
\hline Park.J.Y (stage)2013 & 8 & 99 & 23 & 159 & $4.5 \%$ & $0.52[0.22,1.21]$ & 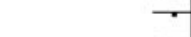 & & \\
\hline Park.J.Y(old) 2012 & 12 & 115 & 30 & 188 & $5.7 \%$ & $0.61[0.30,1.25]$ & & & \\
\hline Subtotal $(95 \% \mathrm{Cl})$ & & 315 & & 555 & $14.5 \%$ & $0.56[0.36,0.90]$ & & & \\
\hline Total events & 27 & & 78 & & & & & & \\
\hline Heterogeneity: $\mathrm{Chi}^{2}=2$ & $50, d f=3$ & $(P=0.4$ & 47); $1^{2}=0$ & & & & & & \\
\hline Test for overall effect: $z$ & $=2.42(\mathrm{P}$ & $=0.02)$ & & & & & & & \\
\hline 10.1.4 urinary-intestin & injury & & & & & & & & \\
\hline Bogani.G 2014 & 1 & 65 & 0 & 65 & $0.1 \%$ & $3.05[0.12,76.17]$ & & & \\
\hline Campos.L.S 2013 & 4 & 16 & 0 & 14 & $0.1 \%$ & $10.44[0.51,213.52]$ & & & \\
\hline Diaz-Feijoo.B 2008 & 0 & 20 & 1 & 30 & $0.3 \%$ & $0.48[0.02,12.37]$ & & & \\
\hline Ditto.A 2015 & 1 & 60 & 0 & 60 & $0.1 \%$ & $3.05[0.12,76.39]$ & & & \\
\hline Frumovitz.M 2007 & 1 & 35 & 1 & 44 & $0.2 \%$ & $1.26[0.08,20.97]$ & & & \\
\hline Ghezzi.F 2007 & 4 & 50 & 3 & 48 & $0.8 \%$ & $1.30[0.28,6.16]$ & & & \\
\hline Kong.T.W 2014 & 3 & 40 & 5 & 48 & $1.2 \%$ & $0.70[0.16,3.12]$ & & & \\
\hline Li.G 2007 & 4 & 90 & 2 & 35 & $0.8 \%$ & $0.77[0.13,4.39]$ & & & \\
\hline Malzoni.M 2009 & 1 & 65 & 1 & 62 & $0.3 \%$ & $0.95[0.06,15.58]$ & & & \\
\hline Nam.J.H 2012 & 17 & 263 & 14 & 263 & $3.7 \%$ & $1.23[0.59,2.55]$ & & & \\
\hline Park.J.K(obese) 2012 & 1 & 54 & 1 & 112 & $0.2 \%$ & $2.09[0.13,34.13]$ & & & \\
\hline Park.J.Y (stage)2013 & 3 & 99 & 1 & 159 & $0.2 \%$ & $4.94[0.51,48.14]$ & & & \\
\hline Park.J.Y(old) 2012 & 5 & 115 & 5 & 188 & $1.0 \%$ & $1.66[0.47,5.88]$ & & & \\
\hline Sobiczewski.P 2009 & 2 & 22 & 2 & 58 & $0.3 \%$ & $2.80[0.37,21.22]$ & & & \\
\hline Taylor.S.E 2011 & 1 & 9 & 0 & 18 & $0.1 \%$ & $6.53[0.24,177.39]$ & & & \\
\hline Uccella.S 2007 & 4 & 50 & 3 & 48 & $0.8 \%$ & $1.30[0.28,6.16]$ & & & \\
\hline Zakashansky.K 2007 & 2 & 30 & 1 & 30 & $0.3 \%$ & $2.07[0.18,24.15]$ & & & \\
\hline Subtotal $(95 \% \mathrm{Cl})$ & & 1083 & & 1282 & $10.5 \%$ & $1.50[0.99,2.26]$ & & 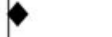 & \\
\hline Total events & 54 & & 40 & & & & & & \\
\hline Heterogeneity: $\mathrm{Chi}^{2}=6$ & $9, d f=16$ & $6(P=0$. & $.98) ; 1^{2}=($ & & & & & & \\
\hline Test for overall effect: 2 & $=1.92(\mathrm{P}$ & $=0.06)$ & & & & & & & \\
\hline Total $(95 \% \mathrm{CI})$ & & 3021 & & 4007 & $100.0 \%$ & $0.82[0.71,0.96]$ & 1 & 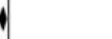 & \\
\hline Total events & 375 & & 607 & & & & & & \\
\hline Heterogeneity: $\mathrm{Chi}^{2}=7$ & $88, \mathrm{df}=4$ & $48(P=0$ & $0.02) ; 1^{2}=$ & $=32 \%$ & & & $.001 \quad 0.1$ & & \\
\hline Test for overall effect: 2 & $=2.46(\mathrm{P}$ & $=0.01)$ & & & & & Favours lap & $\begin{array}{l}10 \\
\text { Favours }\end{array}$ & 1000 \\
\hline
\end{tabular}

FIG. 2. Forest plot and meta-analysis of safety parameters: intraoperative (Intra-op), postoperative (post-op), and perioperative (peri-op) complications and intraoperative urinary-intestine injury. AE, adverse event; CI, confidence interval; lap, laparoscopic; M-H, Mantel-Haenszel method. 
Table 3. Sensitivity Analysis Comparison of Laparoscopic Radical Hysterectomy and AbDominal Radical Hysterectomy

\begin{tabular}{|c|c|c|c|c|c|c|c|c|c|}
\hline \multirow[b]{2}{*}{ Outcomes of interest } & \multirow{2}{*}{$\begin{array}{l}\text { Number of } \\
\text { studies }\end{array}$} & \multicolumn{2}{|c|}{$\begin{array}{l}\text { Number of } \\
\text { patients }\end{array}$} & \multirow[b]{2}{*}{ WMD/OR $(95 \% C I)$} & \multirow[b]{2}{*}{$\mathrm{P}$ value $\mathrm{a}^{\mathrm{a}}$} & \multicolumn{3}{|c|}{$\begin{array}{c}\text { Study } \\
\text { heterogeneity }\end{array}$} & \multirow[b]{2}{*}{$\mathrm{P}$ value } \\
\hline & & $L R H$ & $A R H$ & & & $\chi^{2}$ & df I & $I^{2}(\%)$ & \\
\hline \multicolumn{10}{|l|}{ Primary outcomes } \\
\hline 5-year DFS ${ }^{\mathrm{b}}$ & 6 & 608 & 686 & $0.02(-0.11,0.15)$ & .77 & 4.00 & 5 & 0 & .55 \\
\hline 5-year OS & 4 & 503 & 576 & $-0.01(-0.15,0.14)$ & .93 & 2.81 & 3 & 0 & .42 \\
\hline Recurrence rate ${ }^{b}$ & 7 & 613 & 895 & $0.80(0.53,1.22)$ & .30 & 2.14 & 6 & 0 & .91 \\
\hline \multicolumn{10}{|l|}{ Secondary outcomes } \\
\hline $\begin{array}{l}\text { Intraoperative } \\
\text { bowel-urinary injury }\end{array}$ & 13 & 802 & 889 & $1.49(0.93,2.37)$ & .10 & 5.77 & 12 & 0 & .93 \\
\hline Perioperative complication & 2 & 146 & 255 & $0.66(0.32,1.73)$ & .26 & 1.52 & 1 & 34 & .22 \\
\hline Postoperative complication & 12 & 781 & 1070 & $0.78(0.63,0.97)$ & .02 & 37.14 & 11 & 70 & .0001 \\
\hline Intraoperative complication & 4 & 159 & 175 & $0.79(0.28,2.20)$ & .65 & 3.04 & 3 & 1 & .38 \\
\hline Anorectal recovery (days) & 2 & 73 & 78 & $-0.65(-0.93,-0.36)$ & $<.00001$ & 0.62 & 1 & 0 & .43 \\
\hline Operation time (minutes) & 12 & 656 & 1053 & $20.89(0.53,41.25)$ & .04 & 154.99 & 11 & 93 & $<.00001$ \\
\hline Length of stay (days) & 11 & 757 & 1056 & $-3.98(-4.99,-2.98)$ & $<.00001$ & 73.90 & 10 & 86 & $<.00001$ \\
\hline Blood loss (mL) & 12 & 792 & 1100 & $-179.84(-225.40,-134.28)$ & $<.00001$ & 50.34 & 11 & 78 & $<.00001$ \\
\hline Pelvic LNs removed & 10 & 494 & 793 & $-0.99(-6.07,4.10)$ & .70 & 148.87 & 9 & 94 & $<.00001$ \\
\hline
\end{tabular}

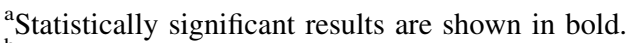

${ }^{\mathrm{b}}$ Prognostic factors were not significantly different.

$\mathrm{ARH}$, abdominal radical hysterectomy; CI, confidence interval; $d f$, degrees of freedom; DFS, disease-free survival; LN, lymph node; LRH, laparoscopic radical hysterectomy; OS, overall survival; WMD/OR, weighted mean difference/odds ratio.

The LRH and ARH groups were not significantly different in terms of survival, and the safety of these approaches was evident. Data included the 5-year DFS and OS, as well as RR, with balanced prognostic factors including lymph node metastasis, Stage IIB or above, non-squamous cancer histology, Grade G3, lymphovascular space invasion, tumor size $\geq 4 \mathrm{~cm}$, positive parametrial margin rate, and positive vaginal margin positive rate, which demonstrated the possibility of recurrence and postoperative adjuvant therapy. The factors assessed were identical, and the surgical specimens removed were similar.

The finding that $\mathrm{LRH}$ and ARH are associated with similar survival indirectly reflects our finding that these approaches have similar safety. The total complication rate was higher in ARH than LRH. These two approaches were associated with similar rates of intraoperative complications, including vessel injury, nerve injury, and thromboembolism. Greater risks of intraoperative bladder and intestine injury were observed in the LRH group, although LRH had longer operative time. Intraoperative urinary injury may be related to several other factors, including surgeons' experience and familiarity with using the laparoscopic system for malignant pelvic conditions. Different postoperative complications such as urinary retention, urinary incontinence, bladder sensation loss, fistula, bowel obstruction, lymphocyst formation, wound dehiscence, hypertrophic scar, and infectious complications were found among the pooled studies. Additionally, LRH is thought to be associated with fewer days of postoperative anorectal function recovery, supporting the use of the minimally invasive laparoscopic approach. In contrast, the postoperative days of catheterization did not significantly differ between these two groups. Conversely, LRH was associated with less blood loss, a lower transfusion rate, and a shorter hospital stay, supporting the use of the minimally invasive LRH approach to treat cervical cancer.
We did not systematically analyze the postoperative pain score. Postoperative pain was assessed during the patients' normal postoperative care and was measured using a 10-point visual analog scale. Three studies reported lower visual analog scale pain scores and less opiate analgesic use in the LRH group than in the ARH group. ${ }^{15,37,38}$ Moreover, quality of life was improved in the LRH group. ${ }^{37}$ The less invasive nature, minimal impairment, and cosmetic satisfaction were the main reasons for the improved quality of life. Psychological fear may increase visual analog scale scores and requests for additional analgesics, as the ARH approach requires a large incision and anesthesia.

For an oncology surgeon, the learning curve for LRH for malignant neoplasm is steeper, so this procedure should be performed by skilled specialists who pay attention to the patients' set-up, port placement, and surgical steps, as these differ from conventional ARH but are required for patient safety and satisfaction (little scarring, fewer hemorrhagic complications, and shorter hospital stays). From this perspective, our findings must be interpreted cautiously because the ability to generalize these results to less experienced surgeons is not guaranteed.

Sensitivity analysis of RCTs and high-quality studies that attained scores of six stars and above was performed to assess the impact of study quality. Outcomes showed that the majority of results were similar and did not significantly differ between the two groups. However, for perioperative complication rates, the sensitivity analysis showed different results. Additional RCTs should be performed with more complete data to allay ethical concerns and patient expectations by providing more definitive conclusions.

This systematic analysis and meta-analysis of LRH and ARH evaluated the safety, efficiency, and clinical complications of the two approaches. Our study had some limitations and bias. The main limitation was that only one RCT 
was included and that the other included studies were retrospective or prospective. One retrospective abstract were included despite lacking complete data. Additionally, a random sequence could not be obtained, which might have introduced bias. Without the full articles, the possibility of selection and data bias increases because of the lack of information for each end point. Furthermore, for DFS and OS, only two studies included HRs, which accurately depict the survival rate, but all included studies had survival curves from which HRs could be calculated. Additionally, the follow-up period was generally short. Thus, long-term outcomes for cervical cancer safety remain unknown. Future systematic reviews should separately evaluate different indications when adequate data are available.

RCTs are controversial because of ethical concerns; thus it is difficult to compare these surgical techniques. This meta-analysis highlights the comparisons and was conducted at an appropriate time, when enough data have accumulated for meta-analytical methods. Additionally, a non-English study ${ }^{37}$ and an article published by Chinese authors ${ }^{25}$ were included to minimize publication bias. All RCT and retrospective articles were reviewed and analyzed. Multiple strategies were identified to control the bias, to evaluate the methodological quality of the studies using strict criteria, and to minimize the heterogeneity with sensitivity analysis. Hence, we provide the most up-to-date information in this area of research.

In conclusion, this article showed that LRH has prognostic results, including DFS, OS, and RR, similar to those of ARH and has fewer complications. The numbers of pelvic and para-aortic lymph nodes removed are identical. LRH shows the nature of minimally invasive nature and little impairment, although LRH is associated with longer operative time and increased risks of intraoperative urinary or intestine injuries. However, the limitations of the included studies prevent us from making more accurate conclusions, and these results must be confirmed and updated by future well-designed RCTs with extensive follow-up periods.

\section{Acknowledgments}

We thank other members of the Department of Gynecologic Oncology at Sun Yat-sen University Cancer Center who did not contribute directly to this review but gave valuable advice throughout the study.

\section{Disclosure Statement}

No competing financial interests exist.

J.L. and T.C. are responsible for study concept and design. T.C. and Q.H. are responsible for acquisition of data. T.C., Y.F., and Q.H. are responsible for analysis and interpretation of data. T.C., Y.F., and J.L. are responsible for drafting of the manuscript. T.W. and Q.H. are responsible for critical revision of the manuscript for important intellectual content. T.C., Y.F., and T.W. are responsible for statistical analysis. T.C., Y.F., and Q.H. are responsible for administrative, technical, or material support. J.L. had full access to all the data and takes responsibility for the integrity of the data and the accuracy of the data analysis so is the guarantor of the content.

\section{References}

1. Renaud MC, Roy M. Laparoscopy in gynaecological oncological surgery in 2005. Rev Gynaecol Perinat Pract 2006; 6:40-46.

2. Tomov S, Gorchev G, Tzvetkov C, Tanchev L, Iliev S. Laparoscopic hysterectomy-Brief history, frequency, indications and contraindications [in Bulgarian]. Akush Ginekol (Sofiia) 2012;51:40-44.

3. Dursun P, Gultekin M, Ayhan A. The history of radical hysterectomy. J Lower Genital Tract Dis 2011;15:235-245.

4. Piver MS, Rutledge F, Smith JP. Five classes of extended hysterectomy for women with cervical cancer. Obstet Gynecol 1974;44:265-272.

5. Clayton RD. Hysterectomy. Best Pract Res Clin Obstet Gynaecol 2006;20:73-87.

6. Roy M, Plante M, Renaud MC. Laparoscopically assisted vaginal radical hysterectomy. Best Pract Res Clin Obstet Gynaecol 2005;19:377-386.

7. Zakashansky K, Bradley WH, Nezhat FR. New techniques in radical hysterectomy. Curr Opin Obstet Gynecol 2008; 20:14-19.

8. Kucukmetin A, Biliatis I, Naik R, Bryant A. Laparoscopically assisted radical vaginal hysterectomy versus radical abdominal hysterectomy for the treatment of early cervical cancer. Cochrane Database Syst Rev 2013;10:CD006651.

9. Liberati A, Altman DG, Tetzlaff J, Mulrow C, Gotzsche PC, Ioannidis JP, Clarke M, Devereaux PJ, Kleijnen J, Moher D. The PRISMA statement for reporting systematic reviews and meta-analyses of studies that evaluate healthcare interventions: Explanation and elaboration. BMJ 2009; 339:b2700.

10. Stroup DF, Berlin JA, Morton SC, Olkin I, Williamson GD, Rennie D, Moher D, Becker BJ, Sipe TA, Thacker SB. Meta-analysis of observational studies in epidemiology: A proposal for reporting. Meta-analysis Of Observational Studies in Epidemiology (MOOSE) Group. JAMA 2000;283: 2008-2012.

11. Phillips B. GRADE: Levels of evidence and grades of recommendation. Arch Dis Child 2004;89:489.

12. Stang A. Critical evaluation of the Newcastle-Ottawa scale for the assessment of the quality of nonrandomized studies in meta-analyses. Eur J Epidemiol 2010;25:603-605.

13. Taggart DP, D'Amico R, Altman DG. Effect of arterial revascularisation on survival: A systematic review of studies comparing bilateral and single internal mammary arteries. Lancet 2001;358:870-875.

14. Hozo SP, Djulbegovic B, Hozo I. Estimating the mean and variance from the median, range, and the size of a sample. BMC Med Res Methodol 2005;5:13.

15. Campos LS, Limberger LF, Stein AT, Kalil AN. Postoperative pain and perioperative outcomes after laparoscopic radical hysterectomy and abdominal radical hysterectomy in patients with early cervical cancer: A randomised controlled trial. Trials 2013;14:293.

16. Taylor SE, McBee WC Jr, Richard SD, Edwards RP. Radical hysterectomy for early stage cervical cancer: Laparoscopy versus laparotomy. JSLS 2011;15:213-217.

17. Frumovitz M, dos Reis R, Sun CC, Milam MR, Bevers MW, Brown J, Slomovitz BM, Ramirez PT. Comparison of total laparoscopic and abdominal radical hysterectomy for patients with early-stage cervical cancer. Obstet Gynecol 2007;110:96-102.

18. Kong TW, Chang SJ, Lee J, Paek J, Ryu HS. Comparison of laparoscopic versus abdominal radical hysterectomy for 
FIGO stage IB and IIA cervical cancer with tumor diameter of $3 \mathrm{~cm}$ or greater. Int J Gynecol Cancer 2014;24:280-288.

19. Park JY, Kim DY, Kim JH, Kim YM, Kim YT, Nam JH. Laparoscopic compared with open radical hysterectomy in obese women with early-stage cervical cancer. Obstet Gynecol 2012;119:1201-1209.

20. Sobiczewski P, Bidzinski M, Derlatka P, Panek G, DanskaBidzinska A, Gmyrek L, Michalski W. Early cervical cancer managed by laparoscopy and conventional surgery: Comparison of treatment results. Int $\mathrm{J}$ Gynecol Cancer 2009;19:1390-1395.

21. Toptas T, Simsek T. Total laparoscopic versus open radical hysterectomy in stage IA2-IB1 cervical cancer: Disease recurrence and survival comparison. J Laparoendosc Adv Surg Tech A 2014;24:373-378.

22. Diaz-Feijoo B, Gil-Moreno A, Perez-Benavente MA, Morchon S, Martinez-Palones JM, Xercavins J. Sentinel lymph node identification and radical hysterectomy with lymphadenectomy in early stage cervical cancer: Laparoscopy versus laparotomy. J Minim Invasive Gynecol 2008; 15:531-537.

23. Ghezzi F, Cromi A, Ciravolo G, Volpi E, Uccella S, Rampinelli F, Bergamini V. Surgicopathologic outcome of laparoscopic versus open radical hysterectomy. Gynecol Oncol 2007;106:502-506.

24. Lee EJ, Kang H, Kim DH. A comparative study of laparoscopic radical hysterectomy with radical abdominal hysterectomy for early-stage cervical cancer: A long-term follow-up study. Eur J Obstet Gynecol Reprod Biol 2011;156:83-86.

25. Li G, Yan X, Shang H, Wang G, Chen L, Han Y. A comparison of laparoscopic radical hysterectomy and pelvic lymphadenectomy and laparotomy in the treatment of Ib-IIa cervical cancer. Gynecol Oncol 2007;105:176-180.

26. Malzoni M, Tinelli R, Cosentino F, Fusco A, Malzoni C. Total laparoscopic radical hysterectomy versus abdominal radical hysterectomy with lymphadenectomy in patients with early cervical cancer: Our experience. Ann Surg Oncol 2009;16:1316-1323.

27. Park JY, Kim DY, Kim JH, Kim YM, Kim YT, Nam JH. Laparoscopic versus open radical hysterectomy for elderly patients with early-stage cervical cancer. Am J Obstet Gynecol 2012;207:195.e1-e8.

28. Park JY, Kim DY, Kim JH, Kim YM, Kim YT, Nam JH. Laparoscopic versus open radical hysterectomy in patients with stage IB2 and IIA2 cervical cancer. J Surg Oncol 2013;108:63-69.

29. Uccella S, Laterza R, Ciravolo G, Volpi E, Franchi M, Zefiro F, Donadello N, Ghezzi F. A comparison of urinary complications following total laparoscopic radical hysterectomy and laparoscopic pelvic lymphadenectomy to open abdominal surgery. Gynecol Oncol 2007;107(1 Suppl 1): S147-S149.

30. Lim YK, Chia YN, Yam KL. Total laparoscopic Wertheim's radical hysterectomy versus Wertheim's radical abdominal hysterectomy in the management of stage I cervical cancer in Singapore: A pilot study. Singapore Med J 2013;54:683-688.

31. Bogani G, Cromi A, Uccella S, Erati M, Casarin J, Pinelli C, Ghezzi F. Laparoscopic versus open abdominal management of cervical cancer: Long-term results from a propensity-matched analysis. J Minim Invasive Gynecol 2014;21:857-862.

32. Ditto A, Martinelli F, Bogani G, Gasparri ML, Di Donato V, Zanaboni F, Lorusso D, Raspagliesi F. Implementation of laparoscopic approach for type B radical hysterectomy: A comparison with open surgical operations. Eur J Surg Oncol 2015;41:34-39.

33. Ghezzi F, Cromi A, Ditto A, Vizza E, Malzoni M, Raspagliesi F, Uccella S, Corrado G, Cosentino F, Gotsch F, Martinelli F, Franchi M. Laparoscopic versus open radical hysterectomy for stage IB2-IIB cervical cancer in the setting of neoadjuvant chemotherapy: A multi-institutional cohort study. Ann Surg Oncol 2013;20:2007-2015.

34. Lin YS. Preliminary results of laparoscopic modified radical hysterectomy in early invasive cervical cancer. J Am Assoc Gynecol Laparosc 2003;10:80-84.

35. Nam JH, Park JY, Kim DY, Kim JH, Kim YM, Kim YT. Laparoscopic versus open radical hysterectomy in earlystage cervical cancer: Long-term survival outcomes in a matched cohort study. Ann Oncol 2012;23:903-911.

36. Zakashansky K, Chuang L, Gretz H, Nagarsheth NP, Rahaman J, Nezhat FR. A case-controlled study of total laparoscopic radical hysterectomy with pelvic lymphadenectomy versus radical abdominal hysterectomy in a fellowship training program. Int J Gynecol Cancer 2007;17: 1075-1082.

37. Stanciu P, Anastasiu DM, Ionescu M, Pantea S, Craina M, Anastasiu D. Laparoscopic radical hysterectomy vs. classical radical hysterectomy. Comparative study of complications and quality of life in patients with early stage cervical cancer [in Italian]. Obstet Ginecol 2013;61: 221-225.

38. Naik R, Jackson KS, Lopes A, Cross P, Henry JA. Laparoscopic assisted radical vaginal hysterectomy versus radical abdominal hysterectomy-A randomised phase II trial: perioperative outcomes and surgicopathological measurements. BJOG 2010;117:746-751.

Address correspondence to: Jihong Liu, MD, PhD Department of Gynecologic Oncology Sun Yat-sen University Cancer Center State Key Laboratory of Oncology in South China Collaborative Innovation Center for Cancer Medicine Guangzhou 510060 People's Republic of China

E-mail: liujh@sysucc.org.cn 